\title{
Fundamentals of Liquid Processing in Low Earth Orbit: From Thermophysical Properties to Microstructure Formation in Metallic Alloys
}

\author{
HANS-JÖRG FECHT ${ }^{1,2}$ and RAINER K. WUNDERLICH ${ }^{1}$ \\ 1.-Institute for Micro and Nanomaterials, Ulm University, Ulm, Germany. 2.-e-mail: \\ hans.fecht@uni-ulm.de
}

\begin{abstract}
Materials science, as an interdisciplinary field with significant attention paid to nanoscience and nanotechnology in recent years, is generally concerned with the synthesis, atomic structure, chemical element distribution and various favorable properties of solid materials and structures. While solid-state processing conditions are generally insensitive to gravity effects, liquid-state processing depends considerably on gravity, leading to strong impact of interfacial phenomena (liquid/solid, liquid/liquid), momentum, heat and mass transport on solidification patterns, as well as further consequences regarding phase selection during solidification and growth conditions, segregation, etc. In this regard, it is interesting to note that almost $100 \%$ of all metallic products are, at some stage, produced through solidification and casting processes. Experiments on metallic liquids excluding the effects of gravity-which is achievable through processing in space, thus reducing gravity effects to microlevels-allow benchmark experiments and the opening of a new realm of experimental possibilities to investigate basic features of the liquid state. The basics and several experimental results are discussed including recent experiments on long time scales under high temperature and containerless processing conditions on board the International Space Station.
\end{abstract}

\section{INTRODUCTION}

Most properties of metals and alloys of industrial use, such as superior mechanical strength, creep resistance at elevated temperatures, improved life times, good ductility, oxidation, corrosion and wear resistance, etc., are determined by their microstructure. Thus, microstructural control during the solidification process is crucial for quality control, waste reduction and the design of new advanced materials for specific technological applications.

Besides the general importance of the thermophysical properties of multi-component alloys for the control of solidification and casting processes, accurate knowledge of such data is also relevant for fundamental science. For example, the analysis of phase transformations, fluid stability and dynamics, fundamental laws like dendrite and pattern formation, as well as fundamental studies of kinetics, transport and pattern formation require the accurate knowledge of the thermophysical properties controlling such processes. ${ }^{1-3}$

In particular, during melt processing of technological relevance, for example in casting, welding, thermal spraying, gas atomization, single crystal growth and directional solidification, crystal nucleation and growth is mainly controlled by the thermophysical properties of the melt. The heat and fluid flow are characterized by dimensionless numbers, such as the Peclét, Prandtl, Rayleigh and Marangoni numbers. Therefore, the basis for understanding and predicting transport phenomena lies in the accurate knowledge of the thermophysical properties which define these dimensionless numbers in the liquid state. However, for most advanced (multicomponent) materials and components of interest, the relevant thermophysical properties needed for the simulation of heat flow and fluid flow during solidification processes are not accurately known 
due to inherent experimental limitations. Over the years, models have been further developed to provide analytical insight but rely on the accuracy (or inaccuracy) of the input data.

Some data can be obtained more or less accurately by conventional methods. High-precision measurements, however, on chemically highly reactive melts and fluids at the temperatures of interest require the application of containerless processing techniques, together with non-contact diagnostic tools. By eliminating the contact between the melt and a crucible, accurate surface nucleation control and the synthesis of materials free of surface contamination and chemical reactions become possible. For metallic melts, electromagnetic levitation is a well-suited and versatile containerless technique. The influence of microgravity conditions on the measurement of certain thermophysical parameters can vary. The reduction of the levitation forces in microgravity leads either to a significant improvement of the accuracy or makes the measurement possible at all.

\section{THEORETICAL ASPECTS}

\section{Scientific Challenges}

On the other hand, the quantitative numerical simulation of casting and solidification processes is increasingly demanded by manufacturers. It provides a rapid tool for the microstructural optimization of high-quality castings, in particular where process reliability and high geometric shape accuracy are important (see e.g., Fig. 1 exhibiting cast structural components and the temperature distribution during casting of a car engine block). Any improvement of numerical simulation results enables further optimization of the defect and grain structure as well as mechanical stress distribution. Through the effective control of unwanted crystallization events, it even becomes possible to produce completely new materials with a controlled amorphous (glassy) or nano-composite structure.

\section{Crystal Nucleation and Growth Transients}

Casting is a non-equilibrium process by which a liquid alloy is solidified. During processing from the melt and cooling below the thermodynamic equilibrium solidification (liquidus) temperature, the first step achieved is the formation of crystalline nuclei of nanometer dimensions that subsequently start to grow. Alternatively, when the formation of nuclei fails, or the growth of nuclei is very sluggish, there is the formation of a metallic glass at the glass transition temperature. At this stage, the main limitations of understanding come from the lack of precise values of the thermophysical properties.

Indeed, the basic understanding of the fundamentals underlying the nucleation of crystals from the melt is in general limited to pure substances under well-controlled conditions. As an example, the nucleation rate $I(T)$ is typically described by ${ }^{4}$

$$
I(T)=\frac{A}{\eta(T)} \exp \left\{-\frac{16 \pi \sigma^{3}}{3 k_{\mathrm{B}} T \Delta G_{V}^{l x}(T)^{2}}\right\}
$$

with $A$ is a prefactor depending mostly on the interaction and wetting behavior of the liquid metal in contact with a container, $\eta(T)$ is the viscosity as a function of temperature, $\sigma$ is the interfacial tension between the liquid and solid phase being formed, and $\Delta G_{V}^{l x}(T)$ is the difference of the Gibbs free energy between the undercooled liquid and the nucleating crystalline phase. For most alloys of actual interest, these thermophysical properties are basically not known. In particular, the interfacial tension between the liquid and crystal to be formed is unknown and usually obtained from nucleation and undercooling experiments, which makes the entire approach rather circular in nature.

Whereas a relatively large set of data exists for low-temperature melts, the data for liquids at high temperatures are rather scarce for pure metals, but are basically non-existent for complex alloys. For example, the reported viscosity measurements generally exhibit a large experimental scatter. Thus, the viscosity data for pure iron-the basis for any steel production-vary by more than $50 \%$ depending on experimental conditions and the sample's purity. Therefore, this nucleation model can describe the basic features but fails to adequately describe the nucleation barrier, in particular for complex multicomponent alloys, which are used for engineering components. Further, the greater importance of diffusion over stirring in the quiescent micro-g environment can dramatically alter the nucleation rate and thus can be used as a benchmark.

\section{Multiphase Growth: Multicomponent Alloys}

The most common case of multiphase growth is certainly the solidification of binary alloys around the eutectic composition, where coupled growth of lamellae or rods of different solid phases is observed. These eutectics are the simplest natural composites, but their wide use, e.g., in electrical devices, is prohibited by the problem of the microstructure defects (e.g., connected lamellae) which seems hard to overcome. Yet, some systems may present interesting mechanical properties, for instance by introducing fragile but high-strength rods into a ductile matrix. ${ }^{3}$

Peritectic systems, in which a second solid phase forms by the reaction between the melt and a first solid phase, are both of practical and fundamental interest. For example, studying the peritectic reaction is taking a step forward towards multicomponent alloys, such as Fe-Cr-Ni steels (D. Matson, this issue) that form more than one solid phase often in a sequence along the solidification path. Besides the technological implications, this is also relevant for 


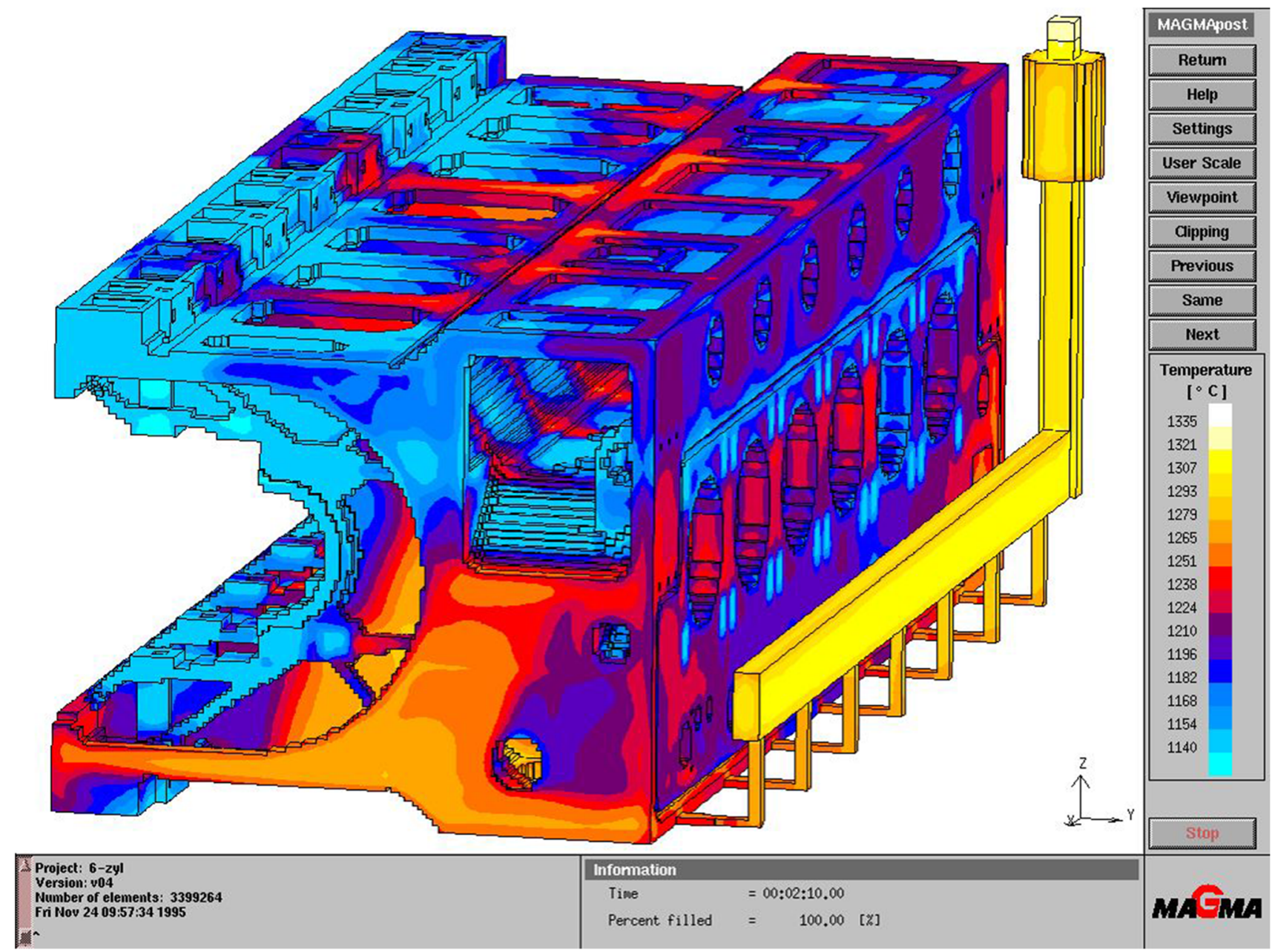

Fig. 1. Fundamental aspects during casting of a complex component of cast iron, here shown in relation to a temperature map predicted for car engine geometry (courtesy MagmaSoft, Germany).

the fundamental understanding of the richness of non-linear dynamic phenomena and requires an exact knowledge of thermophysical property data.

\section{Glass Formation}

If the crystal nucleation rate is sufficiently low, and if the growth of nuclei is sufficiently slow over the entire range of the undercooled liquid below the liquidus temperature, eventually the liquid freezes to a non-crystalline solid, i.e., a glass (Fig. 2). Glasses have been manufactured from silicon and related oxides for thousands of years. More recently, by developing new alloy compositions and processing techniques, it became possible to produce more and more materials in an amorphous form with superior properties in comparison with their (poly)crystalline counterparts.

In particular, new metallic glasses that can be produced in large dimensions and quantitiesso-called bulk metallic glass or supermetals-are emerging as an important industrial and commercial material, superior to conventional Ti-, Al- or Fe-

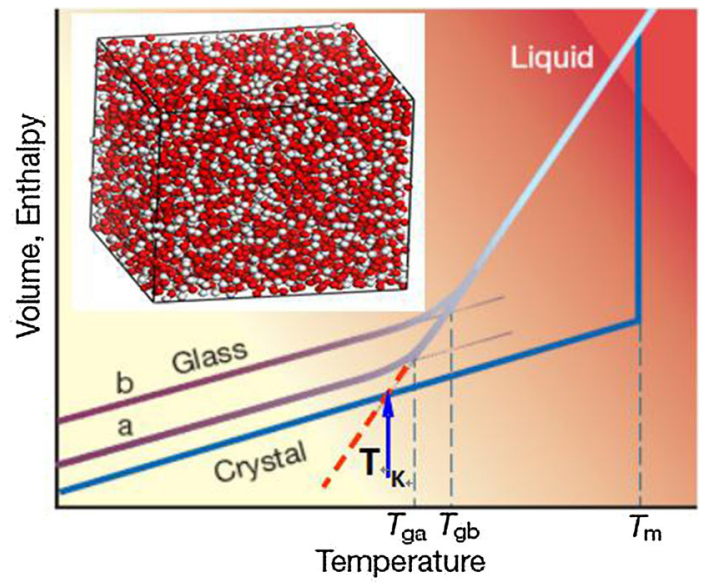

Fig. 2. Volume and enthalpy of a glass-forming alloy as a function of temperature and undercooling (left) and atomic structure in an MD simulation of a Zr-Cu glass (right) (courtesy J. Jiang).

based alloys. They are characterized by about double the mechanical strength (about $2 \mathrm{GPa}$ ) in comparison with conventional materials, excellent 
wear properties and excellent corrosion resistance due to the lack of grain boundaries. These advanced materials can now be produced with dimensions of several $\mathrm{cm}^{3}$ by casting techniques under high vacuum conditions. For a number of pseudo-eutectic $\mathrm{Zr}$-based alloys cooling rates as low as $1 \mathrm{~K} \mathrm{~s}^{-1}$ are sufficient to obtain high levels of undercooling and to produce a bulk glass. ${ }^{5}$

Recent studies indicate a nanoscale chemical segregation accompanying structural ordering in deeply supercooled Cu-Zr-based liquids near the optimum glass-forming composition, and a density anomaly in the supercooled liquid of a $\mathrm{Zr}_{58.5} \mathrm{Nb}_{2.8} \mathrm{Cu}_{15.6} \mathrm{Ni}_{12.8} \mathrm{Al}_{10.3}$ bulk glass that may reflect a fragile/strong transition. ${ }^{6}$ Thermophysical property measurements are needed for clarification and to determine whether similar processes might play a central role in other bulk glass formers.

Due to their strong resistance against crystal nucleation and growth, the control of microstructural development is unique for metallic multicomponent systems with deep eutectics. By a variation in cooling rate and by different heat treatments, the range of microstructural length scales can be varied by several orders of magnitude reaching from regular eutectic microstructures to the nanocrystalline and glassy states.

\section{Magnetohydrodynamic Modeling Aspects}

The experimental techniques to be applied to the materials science problems outlined above are based on containerless processing in an electromagnetic levitation device. In this regard, magnetohydrodynamic modeling MHD, i.e., analyzing the effect of magnetic fields on the internal flow of a levitated hot liquid sample by numerical simulation, will greatly add to the experimental accuracy. The focus here is on the evaluation of the onset of turbulence as a function of material parameters such as the viscosity, density and electrical conductivity, and the inductive heating power input.

\section{EXPERIMENTAL SET-UP}

\section{Microgravity Relevance and Specifics of Low-Gravity Facilities for Metals and Alloys}

The paucity of thermophysical property data for commercial materials as well as materials of fundamental interest is a result of the experimental difficulties generally arising at high temperatures. For chemically non-reactive metals such as, for example, noble metals, some of these data can be obtained more or less accurately by conventional methods. However, high-precision measurements on chemically highly reactive metallic melts, such as $\mathrm{Fe}-$, $\mathrm{Ni}$-, $\mathrm{Ti}-, \mathrm{Zr}$-alloys, etc., require the application of containerless processing techniques and the use of high-precision non-contact diagnostic tools. ${ }^{7}$ For highly reactive metallic melts, electromagnetic levitation (EML) is a well-developed containerless technique which offers several advantages over alternative levitation methods (electrostatic levitation, gas-phase levitation), due to the direct coupling of the high-intensity radio-frequency (rf) electromagnetic field with a metallic sample having an optimum diameter between $6 \mathrm{~mm}$ and $8 \mathrm{~mm}$.

Ground-based experiments using electromagnetic levitation have achieved limited success in measuring the thermophysical properties of liquid alloys, since the high electromagnetic field, $B$, required to lift the sample against gravity (Lorentz force $F \propto \nabla B^{2}$ ) also causes excessive heating and turbulence due to induced eddy currents. In contrast, under microgravity conditions, much smaller levitation forces are needed, since the force of gravity no longer has to be overcome. In fact, in space, only a weak positioning field is required.

The motivation for performing benchmark experiments in the microgravity environment is thus straightforward and at a high level of scientific innovation. Firstly, in space, it is possible to suppress the gravity-induced effects of fluid flow and more subtle sedimentation effects during solidification. Therefore, the contribution to fluid flow and heat transport in the melt can be investigated without the complications of buoyancy-driven thermo-solutal convection and sedimentation/flotation.

Secondly, the space environment on long time scales allows the application of containerless processing techniques, such as electromagnetic levitation. Levitated melts can be controlled effectively at temperatures up to $2200^{\circ} \mathrm{C}$, which in turn enables critical liquid parameters to be measured much more accurately and in a larger temperature range as compared to the earth laboratory.

Experience with parabolic flights (micro-g duration 10-20 s) and TEXUS rocket flights (micro-g duration $180 \mathrm{~s}$ ) already indicated that some aspects of the experiments can be successfully performed, but that micro-g times are far too short to reach thermal equilibrium and measurements in the adiabatic regime. Expanding the experimental time-temperature window through the use of the International Space Station (ISS) will open a completely new realm of space experimentation.

In addition, non-contact measurement methods need to be continuously developed. For surface tension, viscosity, and density measurements, these are based on high-resolution and high-speed optical recording of the sample shape as a function of time and temperature. Calorimetry, including thermal relaxation times, is based on high-resolution noncontact temperature measurement and modulated induction heating with an accuracy of temperature measurements of $<0.1 \mathrm{~K}$. Therefore, it is expected that the specific environment of space and microgravity conditions over long periods of time on board the ISS will lead to breakthroughs in materials science by eliminating complex distorting effects and thus obtaining unambiguous benchmark data and processing conditions. 
For the continued improvement of materials processing with increasing requirements on composition, microstructure and behavior in use, which often implies the breaking of technology barriers, the core of the project is centered on the reliable determination of the thermophysical properties of metallic melts in the high-temperature regime. They are required as input parameters for adequately describing balances in volume phases (heat, chemical species, momentum, etc.) and at boundaries (solid-liquid, liquid-gas, etc.) that together form a set of coupled equations.

New sophisticated methods of controlled levitation, magnetic excitations of surface oscillations, $\mathrm{rf}$ power modulation, optical and temperature diagnostics, and in-time control and data analysis are being developed for the measurement of thermophysical properties. Liquid samples can be processed with a diameter of typically $6.5-8 \mathrm{~mm}$. These methods allow the direct measurement of the following surface- and volume-dependent thermophysical properties in the range between $700^{\circ} \mathrm{C}$ and $2200^{\circ} \mathrm{C}$ as given in Table I.

For the thermophysical property measurements, a variety of alloys were tested including industrial, generic and bulk metallic glass-forming alloys of fundamental interest. The samples selected include:

Ti-Alloys such as Ti6Al4V and Ti-aluminides ${ }^{8}$ as a high-strength light-weight material are of great interest to the aerospace and potentially to the automotive industries. This group of alloys is continuously further developed by the addition of refractory elements such as $\mathrm{Nb}$, Ta and others, which push the liquidus temperature beyond $1700^{\circ} \mathrm{C}$. In this temperature range, no quantitative thermophysical property measurements by conventional methods are possible.

Ni-Based Superalloys These have long since been the workhorse for power generation in land-based turbines and for jet engine propulsion. ${ }^{9}$ Their $\gamma / \gamma^{\prime}$ microstructure is characterized by cubic precipitates of an ordered cubic phase, $\gamma^{\prime}$, in a disordered matrix with a cubic crystal structure of almost identical lattice constants, the $\gamma$-phase, which makes these materials among the highest temperature functional materials in routine service. This basic structure are being continuously further developed, so-called third- and fourth-generation Ni-based alloys.

Fe-Based Alloys Modern steels are used, for example, in land-based turbines for energy production, in jet engines, the automotive industry, advanced fusion concepts and more. They are also very interesting for the study of competing nucleating kinetics of different phases. Steel-casting alloys show complex solidification steps with primary metastable ferritic phase formation, rapidly followed by a transformation to the stable austenite. ${ }^{10}$ This transformation has been shown to be significantly influenced by liquid convection. Maintaining microstructural control requires development of casting models which include these effects.

Bulk Metallic Glasses These represent a new development in materials science. These materials possess superior mechanical properties compared to crystalline conventional materials. ${ }^{11}$ The first generation of these materials was based on combinations of early and late transition metals with further

Table I. Thermophysical properties of levitated liquid samples for ISS experiments and methods applied in MSL-EML

\section{Property}

Enthalpy of fusion/crystallization

Level of undercooling

Specific heat capacity cp $(\mathrm{T})$

Liquid enthalpies, entropies and Gibbs free energies (T)

Thermal diffusivity and conductivity $(\mathrm{T})$

Total hemispherical emissivity $(\mathrm{T})$

Fraction solid/liquid (T)

Surface tension $(\mathrm{T})$

Viscosity $(\mathrm{T})$

Density and coefficient of thermal expansion $(\mathrm{T})$

Electrical conductivity $(\mathrm{T})$

Thermal conductivity check $(\mathrm{T})$

\section{Method}

Temperature-time profile

Temperature-time profile

A.C. modulation calorimetry and measurement of external relaxation times

Integration of $\mathrm{cp}(\mathrm{T}) / \mathrm{Gibb}$ 's thermodynamics

A.C. calorimetry and internal relaxation time plus phase shift between modulated power and temperature signal

A.C. calorimetry and cooling performance A.C. calorimetry in two-phase region

Oscillating drop technique (frequency and amplitude)

Damping of oscillating drop amplitude

Videographic techniques in the coil's axial and radial directions

Inductive method to measure the impedance of a pick-up coil surrounding the sample

Wiedemann-Franz law; valid for solid versus liquid? 


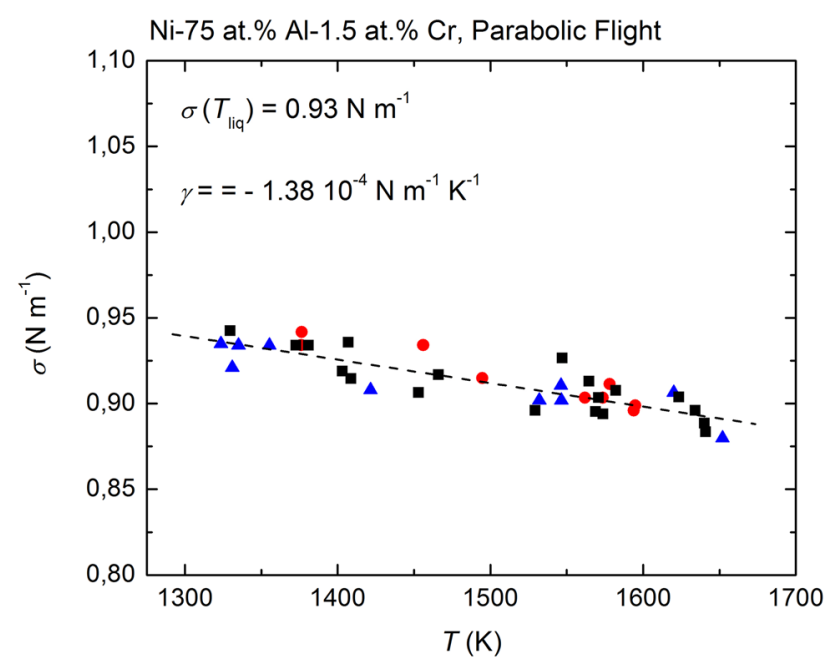

Fig. 3. Surface tension as a function of temperature of a $\mathrm{Ni}$ 75at.\%Al alloy measured with the oscillating drop method in an electromagnetic levitation device on board a parabolic flight airplane (micro-g time about $10 \mathrm{~s}$ ).

addition of, for example, $\mathrm{Al}$ and $\mathrm{B}$. New more lightweight compositions have been developed in recent years. For the improvement of bulk metallic glass formation, a thorough understanding of the kinetic and thermodynamic properties affecting nucleation and phase formation is required, as well as refined models of crystal nucleation from deep undercooling.

\section{RESULTS AND DISCUSSION}

Fresh insight into the stable and metastable undercooled liquid state and metallic alloy solidification can be gained with the potential of engineering novel microstructures. To perform these experiments, it is important to have access to extended periods of reduced gravity. ${ }^{12}$

\section{Parabolic Flights}

Parabolic flights generally provide about $20 \mathrm{~s}$ of reduced gravity of which typically $10 \mathrm{~s}$ are in the liquid state. For materials science experiments at high temperatures, this time is barely sufficient for melting, further heating into the stable liquid, and cooling to solidification of most metallic alloys of interest in a temperature range between $1000^{\circ} \mathrm{C}$ and $2000^{\circ} \mathrm{C}$. Surface oscillations can be excited by a pulse of the heating field, and the surface tension and viscosity are obtained from the oscillation frequency and damping time constant of the oscillations, respectively. Processing must be performed in a gas atmosphere under convective cooling conditions. Under these conditions, however, thermal equilibrium of the melt cannot be reached.

Using the Electromagnetic Levitator, surface oscillations of the liquid hot drop with diameter of $8 \mathrm{~mm}$ can, for instance, be introduced by an electro-

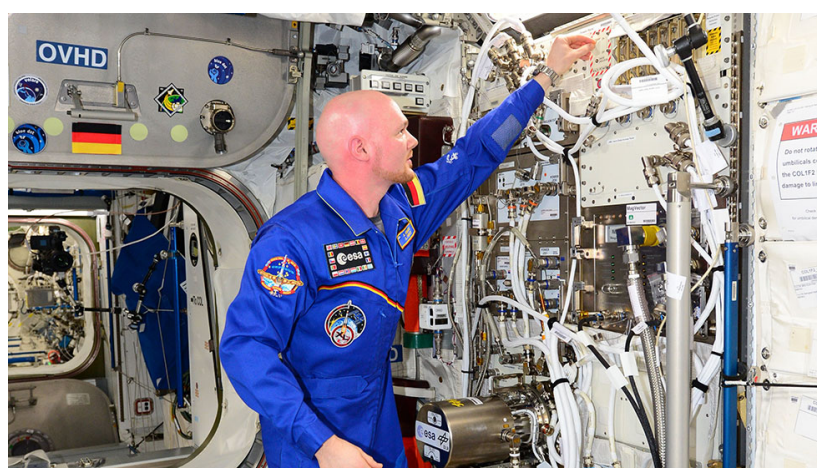

Fig. 4. The electromagnetic levitation device ESL-EML being installed by the astronaut Alexander Gerst in 2014-2015 on the ISS (courtesy European Space Agency).

magnetic pulse and the results analyzed by a highspeed high-resolution video camera. Among the results, Fig. 3 shows the surface tension as a function of temperature in the range $1050-1450^{\circ} \mathrm{C}$ of liquid Ni-75at.\%Al processed under low gravity on four parabolic flights with $10 \mathrm{~s}$ of processing time each.

\section{Sounding Rocket Processing}

TEXUS sounding rockets offer a total of $320 \mathrm{~s}$ of reduced gravity which is typically split between two different experiments. As compared to parabolic flights, the microgravity quality is improved by far. Stable positioning and processing of metallic specimens with widely different electrical resistivity and density was achieved in different sounding rocket flights with an adapted electromagnetic levitation device.

\section{Long-Duration Microgravity Experiments on ISS}

The electromagnetic processing facility MSLEML on the ISS (materials science laboratoryelectromagnetic levitator on the ISS) was installed in 2014-2015. Experiments commenced in 2015 and are planned throughout 2018, possibly until 2024 . In Fig. 4, a photograph of the MSL-EML installed on ISS is shown together with the German astronaut A. Gerst (courtesy ESA). From the list of samples mentioned, two examples will be discussed in the following, namely the Ni-based superalloy LEK94 (Ni-13.8Al-6.6Cr-7.6Co-2.1Ta-1.6W-1Mo1Re-5.6Ta-0.05Hf; MTU, Munich) and the bulk metallic glass-forming alloy VIT106a (Zr-15.6Cu12.8Ni-10.3Al-2.8Nb; Liquidmetal Technologies, Rancho Santa Margarita, CA, USA/Caltech).

As an example of processing for thermophysical property measurements in the MSL-EML, Fig. 5 exhibits the temperature-time profile of the Nibased superalloy LEK94. Processing was under 350 mbar Ar. Measurements needed to be performed in $\mathrm{Ar}$ atmosphere to reduce the evaporation 


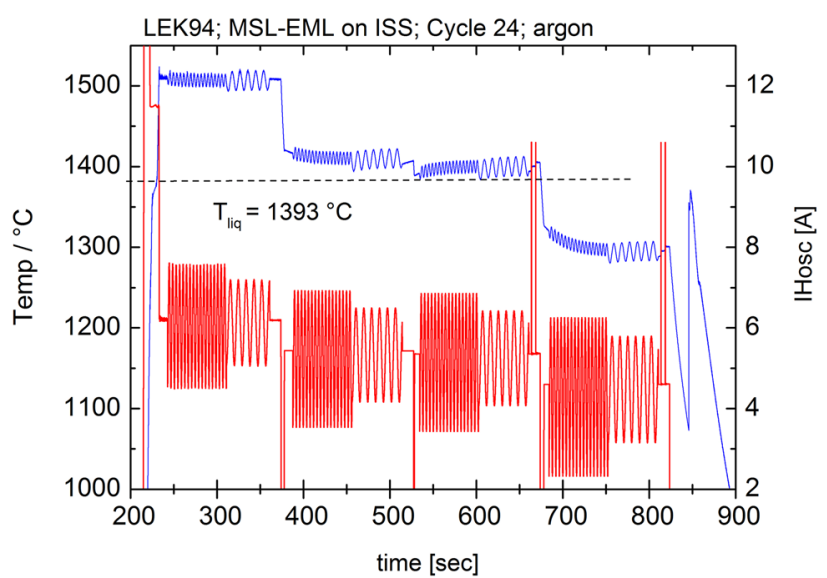

Fig. 5. Temperature-time profile of processing a Ni-based superalloy (LEK94) in the liquid state for thermodynamic property measurements. The programmed rf-heating generator oscillating circuit current (red) on the right hand ordinate and the resulting temperature response (blue) including melting and crystallization responses is shown on the left hand ordinate in the stable and highly undercooled liquid state. The maximum undercooling level is found to be about $300 \mathrm{~K}$ as seen from the recalescence peak at about $850 \mathrm{~s}$ (Color figure online).

of volatile alloy components. The temperature-time profile exhibits the heating in the solid phase, melting and heating in the liquid phase to a preset temperature, followed by stepwise cooling. First, it should be noted that the alloy could be processed in the liquid phase in a stable position for more than $10 \mathrm{~min}$. Moreover, the alloy could be processed in the highly undercooled liquid phase for an extended period of time. The fourth calorimetery measurement was performed at an undercooling of about $100 \mathrm{~K}$. At the end of the nominal processing, the rf heater was turned off, resulting in further undercooling of $\Delta T_{\mathrm{u}} \approx 310 \mathrm{~K}$ followed by recalescence. Such an undercooling behavior over an extended period of time and temperature has not previously been observed for Ni-based superalloys under micro-g conditions. Principally, it reflects the lowimpurity levels of the sample and the excellent processing conditions.

At each temperature plateau, the heating power input was modulated to perform non-contact modulation calorimetry. ${ }^{13}$ Modulation was done with two frequencies, the lower one, $\omega_{1}=0.12 \mathrm{~Hz}$, was chosen to be in the adiabatic regime where the effects of the finite thermal conductivity and radiative and convective heat loss can be neglected, while the larger one, $\omega_{2}=0.28 \mathrm{~Hz}$, is in a regime where internal heat transport contributions to the heat balance are important and can thus be investigated. In combination with magnetohydrodynamic modeling efforts, this may offer a deeper understanding of the internal transport processes in the liquid as a function of temperature and undercooling. The temperature-time profiles were designed such that, before reaching the temperature plateau, a thermal

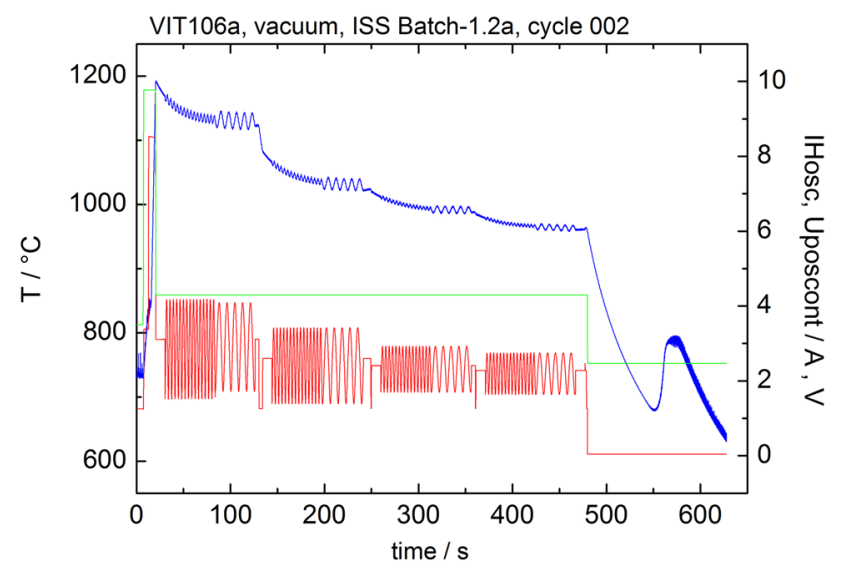

Fig. 6. Temperature-time profile of processing a Zr-based bulk metallic glass-forming alloy (LM VIT106a) in the lower temperature limit of MSL-EML. The rf-heating generator oscillating circuit current (red) on the right hand ordinate and the resulting temperature (blue) is shown on the left hand ordinate. A detailed analysis gives the specific heat, total hemispherical emissivity and thermal conductivity as a function of liquid temperature. The sharp line at ca. $450 \mathrm{~s}$ stems from a magnetic pulse set intentionally to stimulate surface oscillations of the liquid drop for surface tension and viscosity measurements.

transient time is observed in the $\omega_{2}$ modulation signal. The maximum amplitude of the modulation signal of the $\omega_{2}$ modulation component could be identified but needs further analysis. It is further observed that the time-dependence of the modulation maxima is very well represented by a single exponential from which the external relaxation time is evaluated as $\tau_{1}=23.6 \pm 0.1 \mathrm{~s}$. This time constant is characteristic for radiative and conductive heat losses. The strict exponential decay is a result of the linearization of the radiative and conductive boundary condition for relatively small temperature changes.

With these alloys, measurements of the surface tension and viscosity by the oscillating drop method were also performed. The surface oscillation spectra thus obtained exhibited a single peak as expected for a single component liquid and were of excellent quality. An example is the surface oscillation spectrum of a LEK94 following pulse excitation. From the spectrum shown with a center frequency of $34.55 \mathrm{~Hz}$, the surface tension $\sigma$ at $T=1444^{\circ} \mathrm{C}$ is obtained as $\sigma=1.839 \mathrm{~N} \mathrm{~m}^{-1}$. This value is considerably higher than a corresponding value of $\sigma=1.650 \mathrm{~N} \mathrm{~m}^{-1}$ obtained in a recent parabolic flight experiment. The higher value obtained in the ISS experiment is attributed to the very clean processing conditions, including very low $g$-disturbances as exemplified in the unprecedented undercooling behavior and to more quiescent processing conditions with lower cooling rates achieved in the ISS experiments, i.e., $50-60 \mathrm{~K} \mathrm{~s}^{-1}$. For comparison, in parabolic flights, the cooling rate amounts typically to $18-22 \mathrm{~K} \mathrm{~s}^{-1}$. The viscosity at $T_{\text {liq }}$ was obtained from the damping analysis of the decay of 
the oscillation amplitude in ISS experiments as $\eta=8.8 \mathrm{mPa}$ s. The complete evaluation of these experiments is still under way including, in particular, the calibration of the inductive heating power input for quantification of the modulation calorimetry measurements.

As another example, the temperature-time profile of the Zr-based bulk metallic glass-forming alloy VIT106a is shown in Fig. 6. Here, the temperature range of interest was below $1000^{\circ} \mathrm{C}$. The goal of this investigation was an investigation of the thermophysical properties in the stable and undercooled liquid phase, measurements of surface tension and viscosity, and the effect of undercooling and nucleation. This alloy was extensively studied by in situ x-ray scattering in the liquid phase where an obvious structural change in the liquid was observed at about $150 \mathrm{~K}$ above the liquidus temperature. A preliminary evaluation of the specific heat capacity and emissivity data in the liquid phase indicates that such a structural change in the liquid may also be detected in the thermophysics analysis on the ISS.

\section{CONCLUSION}

Experiments under microgravity conditions in the COLUMBUS module on board the ISS offer the ideal conditions to investigate liquid metals and alloys in a wide temperature range typically between $700^{\circ} \mathrm{C}$ and $2100^{\circ} \mathrm{C}$. The recent and exciting results based on A.C. calorimetry and oscillating drop methods clearly demonstrate that the MSLEML instrument on the ISS can be reliably applied to process liquid metallic specimens of a varying composition range over time periods of $10 \mathrm{~min}$ and more for the measurement of thermophysical properties in the stable and widely undercooled liquid phase.

\section{ACKNOWLEDGEMENTS}

The financial support for the this work by the ESA MAP Project Thermoprop AO-99-022 and AO-20091020 Contract No. 4200014306 and by DLR under
Contract 50WM1170 as well as the ESA Project ThermoLab-ISS and ESA-Topical Team on Thermophysical Properties is gratefully acknowledged. Furthermore, the authors are thankful for the active support of the entire science team, flight support by MUSC, DLR Cologne and sample preparation by MTU, Munich, Germany, and Liquidmetal Technologies, Rancho Santa Margarita, CA, USA.

\section{OPEN ACCESS}

This article is distributed under the terms of the Creative Commons Attribution 4.0 International License (http://creativecommons.org/licenses/by/4.0/), which permits unrestricted use, distribution, and reproduction in any medium, provided you give appropriate credit to the original author(s) and the source, provide a link to the Creative Commons license, and indicate if changes were made.

\section{REFERENCES}

1. T. Iida and R.I.L. Guthrie, The Physical Properties of Liquid Metals (Oxford: Clarendon Press, 1988), pp. 168-169.

2. H. Fukuyama and Y. Waseda, eds., High-Temperature Measurements of Materials (New York: Springer, 2009).

3. H.-J. Fecht and B. Billia, ASM Handbook, vol. 22, ed. D. Furrer and S.L. Semiatin (Materials Park: ASM International, 2009), pp. 8-17.

4. K.F. Kelton, Solid State Physics 45, ed. H. Ehrenreich and D. Turnbull (Cambridge: Academic Press, 1991), pp. 75117.

5. H.J. Fecht, J.H. Perepezko, M.C. Lee, and W.L. Johnson, J. Appl. Phys. 68, 4494 (1990).

6. K.F. Kelton, private communication, 12 December 2016.

7. R.K. Wunderlich, D.S. Lee, W.L. Johnson, and H.J. Fecht, Phys. Rev. B 55, 26 (1997).

8. T.J. Nazarova, V.M. Imayev, R.M. Imayev, and H.J. Fecht, Intermetallics 82, 26 (2017).

9. D.U. Furrer and H.J. Fecht, JOM 51, 14 (1999).

10. D.M. Matson, X. Xiao, J.E. Rodriguez, J. Lee, R.W. Hyers, O. Shuleshova, I. Kaban, S. Schneider, C. Karrasch, S. Burggraff, R. Wunderlich, and H.-J. Fecht, JOM (2017). doi:10.1007/s11837-017-2396-5.

11. J.Z. Jiang, D. Hofmann, D.J. Jarvis, and H.J. Fecht, Adv. Eng. Mater. 17, 761 (2014).

12. H.J. Fecht, and B. Billia, in Looking Up: Europe's Quiet Revolution in Microgravity Research (New York: Scientific American, 2008), pp. 68-73.

13. H.J. Fecht and W.L. Johnson, Rev. Sci. Instrum. 62, 1299 (1991). 